\title{
nature
}

nanotechnology

\section{Carbon does it again}

\author{
First isolated less than three years ago, graphene is currently the hottest topic in condensed- \\ matter physics and materials science. Expect further breakthroughs in fundamental physics \\ and, possibly, the advent of graphene-based electronics.
}

Once upon a time, the wonder and diversity of the material world was exemplified by diamond and graphite. How could it be, teachers would ask incredulous students, that these two very different materials - one incredibly beautiful and expensive, the other completely commonplace - were made of the same stuff? And so it remained until the discovery of fullerene molecules, also known as the 'third form of carbon', in 1985.

Carbon nanotubes followed relatively quickly, in 1991, and proved to be even more interesting than fullerenes from both a pure and an applied point of view. Nanotubes were often described as rolled-up sheets of carbon atoms, and these sheets were occasionally referred to as graphene, but no one seemed that interested in working with graphene itself.

All that changed in 2004 when Andre Geim, Kostya Novoselov and coworkers at Manchester University and elsewhere showed that graphene (a.k.a. 'the Mother of all graphitic forms') could be isolated from graphite simply using adhesive tape. They soon discovered that writing on silicon with a piece of graphite was an even better method, while a group at Georgia Tech developed an epitaxial growth process that might be suitable for mass-production ${ }^{1,2}$.

New physics flowed thick and fast: it was soon discovered that the electron mobility of graphene was an order of magnitude higher than that of commercial silicon wafers, which has obvious implications for electronics. Moreover, graphene displays a remarkable range of novel electronic behaviour: in particular, the electrons behave as particles called Dirac fermions that cannot be slowed down because their velocity is independent of their energy (which explains the high mobility). Examples of other novel quantum phenomena - including several that occur at room temperature - are still being discovered ${ }^{3}$.

In recent months, attention has also turned to the structural and mechanical properties of graphene. Researchers in the US have made nanoelectromechanical systems in which the resonator is made of just one or a few graphene sheets ${ }^{4}$, and a European group has shown that single graphene sheets are not perfectly flat ${ }^{5}$.

\section{Graphene displays a remarkable range of novel electronic behaviour.}

The latter result is actually good news because it has been firmly established, by theory and experiment, that perfect two-dimensional crystals cannot exist in a free state.

So what challenges must be overcome before we see 'graphenium inside' ${ }^{1}$ stickers on our laptops? Well the fact that graphene is usually a metal is not an advantage for conventional semiconductor applications, although it is possible to engineer a semiconductor bandgap. However, rather than integrate graphene into conventional silicon devices, a more radical approach would be to make devices directly from graphene: in other words, the conducting channels, quantum dots, interconnects and other elements needed to make truly nanoscale electronic circuits would be cut out from a graphene sheet ${ }^{1}$. Doing this on an industrial scale will require breakthroughs in both epitaxial growth and patterning technology.

There is, of course, nothing new about the promise of novel nanoscale electronics, and graphene has lots of competition in this area. In the short-term, therefore, it is much more likely to have an impact on fundamental physics because, as Dirac fermions, the electrons in graphene do not behave like those in ordinary materials. Using graphene to explore the connections between condensed-matter physics and various fundamental phenomena in quantum electrodynamics is likely to prove highly fruitful ${ }^{6}$.

According to Geim and Novoselov "only the tip of the iceberg has been uncovered so far [and] most experimental groups working on graphene have not published even a single paper on the subject"1. They go on to say that this has been "a truly frustrating experience for theorists", although this has not stopped the latter from publishing papers on everything from Andreev reflection to zitterbewegung (a process that is normally understood in terms of interactions between matter and antimatter). Indeed, like the electrons in graphene, research in the field appears to be unstoppable.

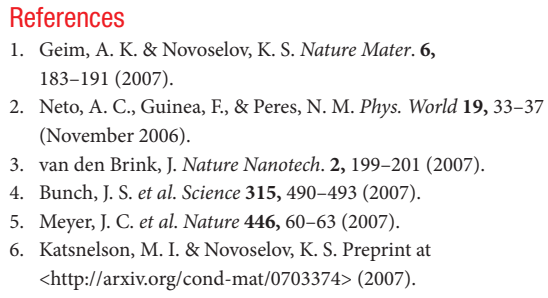

